\title{
COMPUTATION OF KALMAN DECOMPOSITIONS OF PERIODIC SYSTEMS
}

\author{
A. Varga \\ German Aerospace Center, DLR - Oberpfaffenhofen, Institute of Robotics and System Dynamics, D-82234 Wessling, Germany, \\ Andras.Vargaedlr.de
}

Keywords: Periodic systems, discrete-time systems, timevarying systems, Kalman decomposition, numerical methods.

\begin{abstract}
We consider the numerically reliable computation of reachability and observability Kalman decompositions of a periodic system with time-varying dimensions. These decompositions generalize the controllability/observability Kalman decompositions for standard state space systems and have immediate applications in the structural analysis of periodic systems. We propose a structure exploiting numerical algorithm to compute the periodic controllability form by employing exclusively orthogonal similarity transformations. The new algorithm is computationally efficient and strongly backward stable, thus fulfils all requirements for a satisfactory algorithm for periodic systems.
\end{abstract}

\section{Introduction}

Among the important open computational problems which we listed in a recent survey [15], the computation of periodic reachability and observability Kalman decompositions is one which has many useful applications. Besides characterizing the structural properties of the system (reachability/controllability, observability/reconstructibility), properties as stabilizability and detectability can be checked by computing the non-reachable and non-observable characteristic multipliers. Furthermore, by computing the periodic reachability form and the dual periodic observability form of the reachable subsystem, minimal realizations of periodic systems can be easily computed. This computation is a basic step in a recently developed algorithm to evaluate the transfer-function matrix of a periodic system [14].

We consider periodic time-varying systems of the form

$$
\begin{aligned}
x(k+1) & =A_{k} x(k)+B_{k} u(k) \\
y(k) & =C_{k} x(k)
\end{aligned}
$$

where the matrices $A_{k} \in \mathbb{R}^{n_{k+1} \times n_{k}}, B_{k} \in \mathbb{R}^{n_{k+1} \times m_{k}}$, $C_{k} \in \mathbb{R}^{p_{k} \times n_{k}}$, are periodic with period $N \geq 1$. For the definition of the periodic reachability/observability Kalman decompositions it is important to consider the more general case of time-varying dimensions. Note that the Kalman decompositions even of constant dimension periodic systems may lead to reachable/observable and unreachable/unobservable subsystems with time-varying dimensions [5]. Thus, the minimal re- alizations of periodic systems (i.e., reachable and observable) have, in general, time-varying state dimensions [2,3]. Periodic systems with time varying input and output vector dimensions have been considered in [7] and arise in a natural way in some computational problems [14].

Periodic systems with time-varying state dimensions have been already considered earlier in $[6,4]$. However, numerically reliable algorithms for systems with time-varying dimensions have been developed only very recently. Notable examples are the algorithms for the computation of minimal realizations [13], the evaluation of the transfer-function matrix of a periodic system [14], and the numerically stable algorithm to compute the zeros of periodic systems [16]. Note that, the development of general algorithms able to address the case of time-varying dimensions, is one of the requirements which we formulated for a satisfactory numerical algorithm for periodic systems [15].

The computation of Kalman decompositions by using orthogonal similarity transformations was one of the first numerically stable algorithms developed to solve system theoretic problems. In a survey [10], six authors are cited who published in 1981, almost simultaneously, numerically reliable algorithms to compute the controllability Kalman decomposition via the so-called controllability staircase form. Although the corresponding theoretical results have been extended to the periodic case by Grasselli already in 1984 [5], and subsequently have been refined in the works of various authors $[9,4,2]$, until now there exists no computation oriented algorithm to compute the periodic Kalman decompositions.

In this paper, we propose a structure exploiting numerically reliable algorithm to compute the Kalman reachability decomposition for discrete-time periodic systems using exclusively orthogonal similarity transformations. A dual algorithm can be used to compute the Kalman observability decomposition. With these two algorithms, the minimal realization problem of periodic systems can be solved in a numerically reliable way. The new algorithm is computationally efficient and strongly backward stable, thus fulfils all requirements for a satisfactory algorithm for periodic systems.

Notation. For an $N$-periodic matrix $X_{i}$ we use alternatively the script notation

$$
\mathcal{X}:=\operatorname{diag}\left(X_{1}, X_{2}, \ldots, X_{N}\right),
$$

which associates the block-diagonal matrix $\mathcal{X}$ to the cyclic matrix sequence $X_{i}, i=1, \ldots, N$. This notation is consistent with the standard matrix operations as for instance addition, 
multiplication, transposition or inversion. We denote with $\sigma \mathcal{X}$ the $N$-cyclic shift

$$
\sigma \mathcal{X}=\operatorname{diag}\left(X_{2}, \ldots, X_{N}, X_{1}\right)
$$

of the cyclic sequence $X_{k}, k=1, \ldots, N$. By using the script notation, the periodic system (1) will be alternatively denoted by the triple $(\mathcal{A}, \mathcal{B}, \mathcal{C})$. The transition matrix of the system $(1)$ is defined by the $n_{j} \times n_{i}$ matrix $\Phi_{A}(j, i)=A_{j-1} A_{j-2} \cdots A_{i}$, where $\Phi_{A}(i, i):=I_{n_{i}}$. The state transition matrix over one pe$\operatorname{riod} \Phi_{A}(j+N, j) \in \mathbb{R}^{n_{j} \times n_{j}}$ is called the monodromy matrix of system (1) at time $j$ and its eigenvalues are called the characteristic multipliers at time $j$. The set of periodic dimensions $\left\{n_{1}, \ldots, n_{N}\right\}$, will be denoted as $\mathbf{n}$.

\section{Periodic Kalman decompositions}

For the definitions of reachability, observability and minimality of periodic systems we rely on [1] (see also [4] for a more detailed exposition).

Definition 1 The periodic system (1) is reachable at time $k$ if

$$
\operatorname{rank} R_{k}=n_{k},
$$

where $R_{k}$ is the infinite columns reachability matrix

$$
R_{k}=\left[\begin{array}{llll}
B_{k-1} & A_{k-1} B_{k-2} & \cdots & \Phi_{A}(k, i+1) B_{i} \cdots
\end{array}\right] .
$$

The periodic system (1) is completely reachable if (2) holds for all $k$.

Definition 2 The periodic system (1) is observable at time $k$ if

$$
\operatorname{rank} O_{k}=n_{k},
$$

where $O_{k}$ is the infinite rows observability matrix

$$
O_{k}=\left[\begin{array}{c}
C_{k} \\
C_{k+1} A_{k} \\
\vdots \\
C_{i} \Phi_{A}(i, k) \\
\vdots
\end{array}\right] .
$$

The periodic system (1) is completely observable if (4) holds for all $k$.

Definition 3 The periodic system (1) is minimal if it is completely reachable and completely observable.

Let $S_{k} \in \mathrm{R}^{\mathrm{n}_{\mathrm{k}} \times \mathrm{n}_{\mathrm{k}}}$ be an $N$-periodic nonsingular matrix. The reachability, observability and minimality properties are invariant under an $N$-periodic state-space similarity transformation of the form

$$
\widetilde{A}_{k}=S_{k+1}^{-1} A_{k} S_{k}, \quad \widetilde{B}_{k}=S_{k+1}^{-1} B_{k}, \quad \widetilde{C}_{k}=C_{k} S_{k}
$$

The reachability and observability Kalman decompositions of periodic systems have been introduced in [5]. We recall below the main results:
Theorem 1 [5, 7] Every $N$-periodic system $(\mathcal{A}, \mathcal{B}, \mathcal{C})$ is statespace equivalent to an $N$-periodic system $(\widetilde{\mathcal{A}}, \widetilde{\mathcal{B}}, \widetilde{\mathcal{C}})$, where

$$
\widetilde{A}_{k}=\left[\begin{array}{cc}
A_{k}^{r} & * \\
0 & A_{k}^{\bar{r}}
\end{array}\right], \widetilde{B}_{k}=\left[\begin{array}{c}
B_{k}^{r} \\
0
\end{array}\right], \widetilde{C}_{k}=\left[\begin{array}{ll}
C_{k}^{r} & C_{k}^{\bar{r}}
\end{array}\right]
$$

where $A_{k}^{r} \in R^{r_{k+1} \times r_{k}}, r_{k}=\operatorname{rank} R_{k}$ and the pair $\left(\mathcal{A}^{r}, \mathcal{B}^{r}\right)$ is completely reachable.

The decomposition of the system matrices in the form (6) is called the periodic Kalman reachability decomposition (PKRD). In this decomposition, the subsystem $\left(\mathcal{A}^{r}, \mathcal{B}^{r}, \mathcal{C}^{r}\right)$ is completely reachable and the transfer-function matrix (TFM) of the linear time-invariant lifted-representations (see [8]) corresponding to this system and the original system $(\mathcal{A}, \mathcal{B}, \mathcal{C})$ are the same [2]. The unreachable characteristic multipliers of the system (1) are the eigenvalues of $\Phi_{A^{\bar{r}}}(N, 0)$.

Definition 4 The periodic system (1) is completely controllable if all unreachable characteristic multipliers are zero.

The dual result to Theorem 1 is the following one:

Theorem 2 Every $N$-periodic system $(\mathcal{A}, \mathcal{B}, \mathcal{C})$ is state-space equivalent to an $N$-periodic system $(\widetilde{\mathcal{A}}, \widetilde{\mathcal{B}}, \widetilde{\mathcal{C}})$ where

$$
\widetilde{A}_{k}=\left[\begin{array}{cc}
A_{k}^{o} & 0 \\
* & A_{k}^{\bar{o}}
\end{array}\right], \widetilde{B}_{k}=\left[\begin{array}{c}
B_{k}^{o} \\
B_{k}^{\bar{o}}
\end{array}\right], \widetilde{C}_{k}=\left[\begin{array}{ll}
C_{k}^{o} & 0
\end{array}\right]
$$

where $A_{k}^{o} \in R^{q_{k+1} \times q_{k}}$, the pair $\left(\mathcal{A}^{o}, \mathcal{C}^{o}\right)$ is completely observable and $q_{k}=\operatorname{rank} O_{k}$.

The subsystem $\left(\mathcal{A}^{o}, \mathcal{B}^{o}, \mathcal{C}^{o}\right)$ is completely observable and the TFMs of the lifted-representations corresponding to this system and original system $(\mathcal{A}, \mathcal{B}, \mathcal{C})$ are the same [2]. The unobservable characteristic multipliers of the system (1) are the eigenvalues of $\Phi_{A^{\bar{o}}}(N, 0)$.

Definition 5 The periodic system (1) is completely reconstructible if all unobservable characteristic multipliers are zero.

In our developments we use the cyclic lifted system defined in [9] for constant dimensions. Denote by $Z_{\mathbf{n}}$ the cyclic shift matrix

$$
Z_{\mathbf{n}}=\left[\begin{array}{cccc}
0 & \cdots & 0 & I_{n_{N}} \\
I_{n_{1}} & \cdots & 0 & 0 \\
\vdots & \ddots & \vdots & \vdots \\
0 & \cdots & I_{n_{N-1}} & 0
\end{array}\right]
$$

and define (similarly as done in [9] for constant dimensions) the cyclic lifted system, with $\sum_{i=1}^{N} m_{i}$ inputs, $\sum_{i=1}^{N} p_{i}$ outputs, and state vector dimension $\sum_{i=1}^{N} n_{i}$, as the time-invariant system

$$
\begin{aligned}
x_{k}^{C}(h+1) & =F^{C} x_{k}^{C}(h)+G^{C} u_{k}^{C}(h) \\
y_{k}^{C}(h) & =H^{C} x_{k}^{C}(h)
\end{aligned}
$$


where $\left(F^{C}, G^{C}, H^{C}\right):=\left(Z_{\mathbf{n}} \mathcal{A}, Z_{\mathbf{n}} \mathcal{B}, \mathcal{C}\right)$.

The following results are straightforward extensions of those of [9] to the case of time-varying dimensions:

Lemma 1 The pair $(\mathcal{A}, \mathcal{B})$ is completely reachable iff the pair $\left(F^{C}, G^{C}\right)$ is reachable.

Lemma 2 The pair $(\mathcal{A}, \mathcal{C})$ is completely observable iff the pair $\left(F^{C}, H^{C}\right)$ is observable.

\section{PKRD algorithm}

In this section we show that the periodic reachability form (6) can be computed using orthogonal similarity transformations and we develop an efficient computational algorithm which generalizes the algorithm of [11] and similar algorithms cited in [10].

To justify our approach, we consider the periodic pair $(\mathcal{A}, \mathcal{B})$ and let $\mathcal{U}$ be an orthogonal state-space similarity transformation such that each $U_{k+1}$ compresses $B_{k}$ to a full row rank matrix. If $\nu_{k+1}$ is the rank of $B_{k}$, then we can write

$$
U_{k+1}^{T} B_{k}:=\left[\begin{array}{c}
A_{k, 10} \\
0
\end{array}\right] \begin{gathered}
\nu_{k+1} \\
\rho_{k+1}
\end{gathered}
$$

where $A_{k, 10}$ has full row rank. We apply the transformation to $A_{k}$ and partition $U_{k+1}^{T} A_{k} U_{k}$ as follows

$$
U_{k+1}^{T} A_{k} U_{k}:=\left[\begin{array}{cc}
A_{k, 11} & A_{k, 12} \\
\widetilde{B}_{k} & \widetilde{A}_{k} \\
\nu_{k} & \rho_{k}
\end{array}\right] \begin{gathered}
\nu_{k+1} \\
\rho_{k+1}
\end{gathered}
$$

Note that some dimensions can be zero, depending on the ranks of the matrices $B_{k}, k=1, \ldots, N$.

We observe that we can now apply to the reduced pairs a second orthogonal similarity transformation defined by $\mathcal{V}$, with $V_{k}$ of the form $V_{k}=\operatorname{diag}\left(I_{\nu_{k}}, \widetilde{U}_{k}\right)$. These transformations will affect only $\widetilde{B}_{k}, \widetilde{A}_{k}$ and $A_{k, 12}$. Now we choose $\widetilde{U}_{k+1}$ to compress the rows of $\widetilde{B}_{k}$ to a full row rank matrix and repeat the partitioning in form (9) and (10) for the matrices $\widetilde{U}_{k+1}^{T} \widetilde{B}_{k, 1}$ and $\widetilde{U}_{k+1}^{T} \widetilde{A}_{k} \widetilde{U}_{k}$. We obtain globally

$$
V_{k+1}^{T} U_{k+1}^{T} A_{k} U_{k} V_{k}:=\left[\begin{array}{ccc}
A_{k, 11} & A_{k, 12} & A_{k, 13} \\
A_{k, 21} & A_{k, 22} & A_{k, 23} \\
0 & \widehat{B}_{k} & \widetilde{A}_{k}
\end{array}\right] \tilde{\nu}_{k+1} \tilde{\rho}_{k+1}
$$

where some submatrices have been redefined. This reduction process continues until $\tilde{\nu}_{k}=0$ for $k=1, \ldots, N$, that is, all $\widehat{B}_{k}=0$, or $\tilde{\rho}_{k}=0$, for $k=1, \ldots, N$, that is all $\widehat{B}_{k}$ have full row rank.
The following implementable algorithm formalizes the above ideas:

\section{PKRD Algorithm: Periodic Kalman Reachability Decom- position}

Given $A_{k} \in \mathbb{R}^{n_{k+1} \times n_{k}}, B_{k} \in \mathbb{R}^{n_{k+1} \times m_{k}}$ and $C_{k} \in \mathbb{R}^{p_{k} \times n_{k}}$ for $k=1, \ldots, N$, this algorithm computes the orthogonal matrices $Q_{k}, k=1, \ldots, N$, such that the transformed system $\left(\sigma \mathcal{Q}^{T} \mathcal{A} \mathcal{Q}, \sigma \mathcal{Q}^{T} \mathcal{B}, \mathcal{C} \mathcal{Q}\right)$ is in the periodic Kalman reachability form (6).

1. Set $j=1$ and $r_{k}=0, \nu_{k}^{(0)}=m_{k}, A_{k}^{(0)}=A_{k}, B_{k}^{(0)}=$ $B_{k}, Q_{k}=I_{n_{k}}$ for $k=1, \ldots, N$.

2. For $k=1, \ldots, N$, compute the orthogonal matrix $U_{k+1}$ to compress the matrix $B_{k}^{(j-1)} \in \mathbb{R}^{\left(n_{k+1}-r_{k+1}\right) \times \nu_{k}^{(j-1)}}$ to a full row rank matrix

$$
U_{k+1}^{T} B_{k}^{(j-1)}:=\left[\begin{array}{c}
A_{k ; j, j-1} \\
0 \\
\nu_{k}^{(j-1)}
\end{array}\right] \begin{gathered}
\nu_{k+1}^{(j)} \\
\rho_{k+1}^{(j)}
\end{gathered}
$$

3. For $k=1, \ldots, N$, compute $U_{k+1}^{T} A_{k}^{(j-1)} U_{k}$ and partition it in the form

$$
U_{k+1}^{T} A_{k}^{(j-1)} U_{k}:=\left[\begin{array}{cc}
A_{k ; j, j} & A_{k ; j, j+1} \\
B_{k}^{(j)} & A_{k}^{(j)}
\end{array}\right] \nu_{k+1}^{(j)} \rho_{k+1}^{(j)}
$$

4. For $k=1, \ldots, N$ and $i=1, \ldots, j-1$, compute

$$
\begin{aligned}
A_{k ; i, j} U_{k}:= & \left.\begin{array}{cc}
A_{k ; i, j} & A_{k ; i, j+1}
\end{array}\right] \\
\nu_{k}^{(j)} & \rho_{k}^{(j)}
\end{aligned}
$$

5. For $k=1, \ldots, N$, compute $Q_{k} \leftarrow Q_{k} \operatorname{diag}\left(I_{r_{k}}, U_{k}\right)$, $C_{k} \leftarrow C_{k} \operatorname{diag}\left(I_{r_{k}}, U_{k}\right)$.

6. $r_{k} \leftarrow r_{k}+\nu_{k}^{(j)}$, for $k=1, \ldots, N$;

$$
\text { if } \rho_{k}^{(j)}=0 \text { for } k=1, \ldots, N \text {, then } \ell=j \text {, Exit } 1 .
$$

7. If $\nu_{k}^{(j)}=0$ for $k=1, \ldots, N$, then $\ell \leftarrow j-1$, Exit 2; else, $j \leftarrow j+1$ and go to Step 2 .

After performing the PKRD Algorithm, each pair $\left(A_{k}, B_{k}\right)$ is in the periodic reachability form (6), where the pair $\left(A_{k}^{r}, B_{k}^{r}\right)$ is in a staircase form

$$
\left[B_{k}^{r} \mid A_{k}^{r}\right]=\left[\begin{array}{c|cccc}
A_{k ; 1,0} & A_{k ; 1,1} & A_{k ; 1,2} & \ldots & A_{k ; 1, \ell} \\
O & A_{k ; 2,1} & A_{k ; 2,2} & \ldots & A_{k ; 2, \ell} \\
\vdots & \vdots & \ddots & \ddots & \vdots \\
O & O & O & A_{k ; \ell, \ell-1} & A_{k ; \ell, \ell}
\end{array}\right]
$$

and $A_{k}^{\bar{r}} \in \mathbb{R}^{\rho_{k+1}^{(\ell)} \times \rho_{k}^{(\ell)}}$. In (12), $A_{k ; i, i} \in \mathbb{R}^{\nu_{k+1}^{(i)} \times \nu_{k}^{(i)}}, i=$ $1, \ldots, \ell ; A_{k ; i, i-1} \in \mathbb{R}^{\nu_{k+1}^{(i)} \times \nu_{k}^{(i-1)}}$ and $\operatorname{rank} A_{k ; i, i-1}=\nu_{k+1}^{(i)}$ $i=1, \ldots, \ell$.

We have the following important result. 
Theorem 3 For each pair $(\mathcal{A}, \mathcal{B})$ there exists an orthogonal matrix $\mathcal{Q}$ such that the transformed pair $\left(\sigma \mathcal{Q}^{T} \mathcal{A} \mathcal{Q}, \sigma \mathcal{Q}^{T} \mathcal{B}\right)$ is in the periodic reachability form (6).

Proof. We apply the PKRD Algorithm to the pair $(\mathcal{A}, \mathcal{B})$ (assuming $\mathcal{C}=0$ ) and obtain $\mathcal{Q}$ such that the pair $\left(\sigma \mathcal{Q}^{T} \mathcal{A} \mathcal{Q}, \sigma \mathcal{Q}^{T} \mathcal{B}\right)$ is in the form (6) with the pair $\left(\mathcal{A}^{r}, \mathcal{B}^{r}\right)$ in the form (12). We need to show that this pair is reachable. Consider the pencil $\left[Z_{\mathbf{r}} \mathcal{A}^{r}-z I \quad Z_{\mathbf{r}} \mathcal{B}^{r}\right]$ of the cyclic lifted system (see (8)) corresponding to the pair $\left(\mathcal{A}^{r}, \mathcal{B}^{r}\right)$. According to Lemma 1 and the Popov-Belevich-Hautus test, to prove reachability we need only to show that this pencil has full row rank $\sum_{k=1}^{N} r_{k}$ for all $z \in \mathbb{C}$.

By column and row permutations we can bring this pencil in the form

$$
R(z)=\left[\begin{array}{ccccc}
S_{1} & -z T_{1} & O & \cdots & O \\
O & S_{2} & -z T_{2} & \cdots & O \\
\vdots & \ddots & \ddots & \ddots & \vdots \\
O & & & S_{N-1} & -z T_{N-1} \\
-z T_{N} & O & \cdots & O & S_{N}
\end{array}\right]
$$

where, for $k=1, \ldots, N$,

$$
S_{k}:=\left[\begin{array}{ll}
B_{k}^{r} & A_{k}^{r}
\end{array}\right], \quad T_{k}:=\left[\begin{array}{ll}
O & I_{r_{k+1}}
\end{array}\right]
$$

Note that by construction, each $S_{k}$ has full row rank $r_{k+1}=$ $\sum_{i=1}^{\ell} \nu_{k+1}^{(i)}$. Thus, by performing $\ell$ cyclic column reductions using elementary column operations, we can bring $R(z)$, for any finite $z$, in the form $\operatorname{diag}\left(S_{1}, \ldots, S_{N}\right)$. However, this matrix has full row rank, because, each $S_{k}$ has full row rank.

The first $r_{k}$ columns of $Q_{k}$ form an orthonormal basis for the rechability subspace $\operatorname{Im} R_{k}$. For each pair $\left(A_{k}^{r}, B_{k}^{r}\right)$ we can define a reachability index $\mu_{k}$ as the largest value of $i$ such that $\nu_{k}^{(i)} \neq 0$. The periodic system (1) is reachable at time $k$ if $r_{k}=n_{k}$.

Remark 1. Let $h_{k}$ be the least integer such that $\nu_{k+1}^{\left(h_{k}\right)}=$ 0 . Then, it is easy to see that the trailing $\sum_{i=h_{k}}^{\ell} \nu_{k+1}^{(i)} \times$ $\sum_{i=h_{k}}^{\ell} \nu_{k}^{(i)}$ block of $A_{k}^{r}$ is in a block upper trapezoidal form with all diagonal blocks having full row rank. It follows that the resulting matrices $\widetilde{B}_{k}$ and $\widetilde{A}_{k}$ of the PKRD (6) have, in general, the forms

$$
\begin{gathered}
\widetilde{B}_{k}=\left[\begin{array}{c}
B_{k}^{r} \\
\hline 0
\end{array}\right]=\left[\begin{array}{c}
B_{k, 1}^{r} \\
0 \\
\hline 0
\end{array}\right], \\
\widetilde{A}_{k}=\left[\begin{array}{c|c}
A_{k}^{r} & * \\
\hline 0 & A_{k}^{r}
\end{array}\right]=\left[\begin{array}{cc|c}
A_{k, 11}^{r} & A_{k, 12}^{r} & * \\
0 & A_{k, 22}^{r} & * \\
\hline 0 & 0 & A_{k}^{r}
\end{array}\right],
\end{gathered}
$$

where $A_{k, 22}^{r}$ has full row rank. Note that in the single-input case, the leading block $A_{k, 11}^{r}$ is in an unreduced upper Hessenberg form, while the trailing block $A_{k, 22}^{r}$ is full row rank upper trapezoidal.
Remark 2. It is possible to further reduce $A_{k}^{\bar{r}}$ by separating the zero and nonzero characteristic multipliers in the product $\Phi_{A^{\bar{r}}}(N, 0)$. This can be done once again by employing exclusively orthogonal transformations. The resulting periodic matrix after this separation has the form

$$
A_{k}^{\bar{r}}=\left[\begin{array}{cc}
A_{k}^{0} & * \\
0 & A_{k}^{\bar{c}}
\end{array}\right]
$$

where all characteristic values of the periodic matrix $A_{k}^{0}$ are zero, and the periodic matrix $A_{k}^{\bar{c}} \in \mathbb{R}^{n_{\bar{c}} \times n_{\bar{c}}}$ has constant dimension, is square and nonsingular. The eigenvalues of $\Phi_{A_{\bar{c}}}$ represents the uncontrollable characteristic multipliers of the periodic system (1). It follows that the periodic system (1) is stabilizable if all uncontrollable characteristic multipliers lie in the interior of the unit circle.

Remark 3. The PKRD Algorithm can be extended to periodic descriptor systems of the form

$$
\begin{aligned}
E_{k} x(k+1) & =A_{k} x(k)+B_{k} u(k) \\
y(k) & =C_{k} x(k)
\end{aligned}
$$

where the matrices $A_{k}, B_{k}$, and $C_{k}$ are the same as in (1) and $E_{k} \in \mathbb{R}^{n_{k+1} \times n_{k+1}}$ is an $N$-periodic invertible matrix. The similarity transformation used in this case has the form

$$
\begin{array}{ll}
\widetilde{E}_{k}=T_{k+1} E_{k} S_{k+1}, & \widetilde{A}_{k}=T_{k+1} A_{k} S_{k}, \\
\widetilde{B}_{k}=T_{k+1} B_{k}, & \widetilde{C}_{k}=C_{k} S_{k}
\end{array}
$$

with $S_{k}$ and $T_{k} N$-periodic nonsingular matrices.

After a preliminary reduction of $E_{k}$ to an upper triangular form using suitable orthogonal $S_{k}$ and $T_{k}$, we perform, as in the PKRD Algorithm, the row compression on $B_{k}$ using an orthogonal transformation $U_{k+1}$. The only difference in the descriptor case is that the upper triangular form of $E_{k}$ is preserved while reducing $B_{k}$. This can be done by computing an appropriate $V_{k+1}$ such that $U_{k+1}^{T} E_{k} V_{k+1}$ remains upper triangular. In fact, the compression of $B_{k}$ and maintaining the upper triangular form of $E_{k}$ can be done simultaneously, in a similar way as done in [12] for standard descriptor systems. The combined reduction and restoring of triangular form can efficiently be done by employing orthogonal Givens transformations.

\section{Numerical aspects}

To estimate the necessary floating-point operations (flops) necessary to compute the periodic reachability Kalman decomposition, we assume for simplicity constant dimensions: $n=n_{k}$, $m=m_{k}, p=p_{k}$. The worst-case operations count result if the periodic system is reachable. In this case, if we use Householder transformations based QR decompositions with column pivoting for the row compressions in the PKRD Algorithm, then we can easily give an estimate of the total number of flops necessary to compute the PKRD as

$$
N_{\text {flops }}=N\left(\frac{5}{3} n^{3}+(p+m) n^{2}\right)
$$


To accumulate the transformations the algorithm needs additionally $\mathrm{Nn}^{3}$ flops. Thus, the computational complexity of this algorithm is $O\left(\mathrm{Nn}^{3}\right)$. The same computational complexity can be achieved also in the descriptor case.

All computations can be performed in place, thus the required memory of $(n+m+p) n N$ storage locations is minimal if the transformations are not accumulated. The information on the performed transformations can be compactly stored in the generated zero submatrices during the reduction, and in additional $N n$-vectors. To form the transformation matrices explicitly, $N n^{2}$ additional storage locations are necessary. These figures are valid also for time-varying dimensions, where $n, m$ and $p$ are now the maximum values of state, input and output vector dimensions, respectively.

The strong backward stability of the PKRD Algorithm can be easily proved. The basic idea is that each transformation $U_{k}$ can be computed and applied in a numerically stable way. A sequence of such transformations can be also performed in a numerically stable way, since each orthogonal matrix has unity norm. For details see [17]. It follows that the results computed with the PKRD Algorithm are exact for slightly perturbed initial matrices $\bar{A}_{k}, \bar{B}_{k}, \bar{C}_{k}$, which satisfy

$$
\|\bar{X}-X\| \leq \varepsilon_{X}\|X\|, \quad X=A_{k}, B_{k}, C_{k}
$$

where, in each case, $\varepsilon_{X}$ is a modest multiple of the relative machine precision $\varepsilon_{M}$.

\section{Applications}

\subsection{Computation of PKOD}

To compute the PKOD, an PKOD Algorithm analogous PKRD Algorithm can be devised. Instead row compressions, this algorithm performs column compressions on the matrices $C_{k}^{(i)}$ in the successively generated pairs $\left(A_{k}^{(i)}, C_{k}^{(i)}\right)$. The resulting algorithm can be seen as the application of the PKRD Algorithm to a certain dual periodic system. The following procedure formalizes the main steps of this approach:

\section{PKOD Algorithm: Periodic Kalman Observability Decom- position}

Given $A_{k} \in \mathbb{R}^{n_{k+1} \times n_{k}}, B_{k} \in \mathbb{R}^{n_{k+1} \times m_{k}}$ and $C_{k} \in \mathbb{R}^{p_{k} \times n_{k}}$ for $k=1, \ldots, N$, this algorithm computes the orthogonal matrices $Q_{k}, k=1, \ldots, N$, such that the transformed system $\left(\sigma \mathcal{Q}^{T} \mathcal{A} \mathcal{Q}, \sigma \mathcal{Q}^{T} \mathcal{B}, \mathcal{C} \mathcal{Q}\right)$ is in the periodic Kalman observability form (7).

1. For $k=1, \ldots, N$, form the dual system matrices

$$
\widehat{A}_{k}=A_{N-k+1}^{T}, \quad \widehat{B}_{k}=C_{N-k+1}^{T}, \quad \widehat{C}_{k}=B_{N-k+1}^{T}
$$

2. Apply the PKRD Algorithm to the triple $(\widehat{\mathcal{A}}, \widehat{\mathcal{B}}, \widehat{\mathcal{C}})$ to determine the orthogonal $\mathrm{N}$-periodic transformation matrices $\widehat{Q}_{k}$ such that

$$
\widehat{\mathcal{A}} \leftarrow \sigma \widehat{\mathcal{Q}}^{T} \widehat{\mathcal{A}} \widehat{\mathcal{Q}}, \quad \widehat{\mathcal{B}} \leftarrow \sigma \widehat{\mathcal{Q}}^{T} \widehat{\mathcal{B}}, \quad \widehat{\mathcal{C}} \leftarrow \widehat{\mathcal{C}} \widehat{\mathcal{Q}}
$$

with the resulting pair $(\widehat{\mathcal{A}}, \widehat{\mathcal{B}})$ in the periodic reachability form

$$
\widehat{A}_{k}=\left[\begin{array}{cc}
\widehat{A}_{k}^{r} & * \\
0 & \widehat{A}_{k}^{\bar{r}}
\end{array}\right], \quad \widehat{B}_{k}=\left[\begin{array}{c}
\widehat{B}_{k}^{r} \\
0
\end{array}\right]
$$

3. For $k=1, \ldots, N$, form the system matrices of the PKOD

$$
A_{k} \leftarrow \widehat{A}_{N-k+1}^{T}, \quad B_{k} \leftarrow \widehat{C}_{N-k+1}^{T}, \quad C_{k} \leftarrow \widehat{B}_{N-k+1}^{T}
$$

4. Set $Q_{N}=\widehat{Q}_{N}$, and $Q_{k}=\widehat{Q}_{N-k}$, for $k=1, \ldots, N-1$.

Using the computed results of this algorithm, the reconstructibility and detectability properties can be analyzed in a similar way as indicated in Remark 2 for the dual properties of controllability and stabilizability, respectively.

\subsection{Computation of minimal realizations}

The numerical computation of minimal realizations of periodic systems has been addressed in [13], where a balancing-related approach was proposed. This algorithm relies on the computation of the extended periodic Schur form of $A_{k}$, and involves the solution of two non-negative definite periodic Lyapunov equations. This algorithm is numerically reliable, since each computational step relies on strongly backward stable algorithms. The main advantage of this algorithm is that the $N$ rank decisions necessary to obtain the state-vector dimensions of a minimal realization are performed only once at the end of the algorithm. Thus, this approach is very reliable in determining the order of the minimal realizations.

In some applications, as for example when computing the TFM of a periodic system [14], the algorithmic efficiency aspects play an important role. Thus, instead employing the above algorithm as proposed in [14], we can alternatively use a significantly more efficient procedure to compute minimal realizations by eliminating successively the unreachable and unobservable parts. A two step procedure is formalized below:

\section{Minimal realization procedure}

1. Apply the PKRD Algorithm to the system $(\mathcal{A}, \mathcal{B}, \mathcal{C})$ to compute the reachable realization $\left(\mathcal{A}^{r}, \mathcal{B}^{r}, \mathcal{C}^{r}\right)$.

2. Apply the PKOD Algorithm to the reachable system $\left(\mathcal{A}^{r}, \mathcal{B}^{r}, \mathcal{C}^{r}\right)$ to compute the minimal realization as the observable part $\left(\mathcal{A}^{\text {ro }}, \mathcal{B}^{\text {ro }}, \mathcal{C}^{\text {ro }}\right)$.

This algorithm is strongly backward stable and has a computational complexity of $O\left(N n^{3}\right)$. 


\section{Numerical example}

Consider the 3-periodic single-input single-output system with the constant dimension system matrices

$$
\begin{aligned}
& A_{1}=\left[\begin{array}{ll}
0 & 1 \\
0 & 0
\end{array}\right], B_{1}=\left[\begin{array}{l}
3 \\
0
\end{array}\right], C_{1}=\left[\begin{array}{ll}
0 & 1
\end{array}\right] \\
& A_{2}=\left[\begin{array}{ll}
1 & 2 \\
0 & 0
\end{array}\right], B_{2}=\left[\begin{array}{l}
0 \\
1
\end{array}\right], C_{2}=\left[\begin{array}{ll}
2 & 4
\end{array}\right] \\
& A_{3}=\left[\begin{array}{ll}
0 & 0 \\
1 & 4
\end{array}\right], B_{3}=\left[\begin{array}{l}
0 \\
1
\end{array}\right], C_{3}=\left[\begin{array}{ll}
3 & 1
\end{array}\right]
\end{aligned}
$$

By applying the PKRD Algorithm with

$$
Q_{1}=\left[\begin{array}{ll}
1 & 0 \\
0 & 1
\end{array}\right], \quad Q_{2}=\left[\begin{array}{ll}
0 & 1 \\
1 & 0
\end{array}\right], \quad Q_{3}=\left[\begin{array}{ll}
0 & 1 \\
1 & 0
\end{array}\right]
$$

we obtain the periodic matrices in the reachability form

$$
\begin{aligned}
& {\left[\begin{array}{c|c}
A_{1}^{r} & * \\
\hline 0 & *
\end{array}\right]=\left[\begin{array}{c|c}
1 & 0 \\
\hline 0 & 0
\end{array}\right],\left[\begin{array}{c}
B_{1}^{r} \\
\hline 0
\end{array}\right]=\left[\frac{3}{0}\right],\left[C_{1}^{r} \mid *\right]=[1 \mid 0]} \\
& {\left[A_{2}^{r} \mid *\right]=\left[\begin{array}{l|l}
0 & 0 \\
1 & 2
\end{array}\right], \quad B_{2}^{r}=\left[\begin{array}{l}
1 \\
0
\end{array}\right], \quad\left[C_{2}^{r} \mid *\right]=[2 \mid 4]} \\
& {\left[\frac{A_{3}^{r}}{0}\right]=\left[\begin{array}{ll}
4 & 1 \\
\hline 0 & 0
\end{array}\right], \quad\left[\frac{B_{3}^{r}}{0}\right]=\left[\frac{1}{0}\right], C_{3}^{r}=\left[\begin{array}{ll}
1 & 3
\end{array}\right]}
\end{aligned}
$$

Thus, the reachable part has time-varying state dimensions, $r_{1}=1, r_{2}=1, r_{3}=2$. Note that this part is also observable, thus $\left(\mathcal{A}^{r}, \mathcal{B}^{r}, \mathcal{C}^{r}\right)$ represents a minimal realization of the original system.

\section{Conclusion}

In this paper we proposed a strongly backward stable algorithm to compute the periodic reachability Kalman decomposition of a periodic system. This algorithm can be applied to compute the periodic observability Kalman decomposition as well, and thus can be used to compute minimal realizations of periodic systems. The algorithm works for system matrices with timevarying dimensions and can be easily extended to descriptor periodic systems as well. By fully exploiting the problem structure, an acceptable computational complexity can be achieved, which is linear in the period $N$ and cubic in the maximum dimension of state vector. Thus, the new algorithm fulfils all requirements which we formulated in [15] for a satisfactory algorithm for periodic systems.

\section{References}

[1] S. Bittanti and P. Colaneri. Analysis of discrete-time linear periodic systems. In Digital Control and Signal Processing Systems and Techniques, (C. T. Leondes, Ed.), vol. 78 of Control and Dynamics Systems, pp. 313-339., Academic Press, 1996.

[2] P. Colaneri and S. Longhi. The realization problem for linear periodic systems. Automatica, 31:775-779, 1995.
[3] I. Gohberg, M. A. Kaashoek, and J. Kos. Classification of linear periodic difference equations under periodic or kinematic similarity. SIAM J. Matrix Anal. Appl., 21:481507, 1999.

[4] I. Gohberg, M. A. Kaashoek, and L. Lerer. Minimality and realization of discrete time-varying systems. Operator Theory: Advances and Applications, 56:261-296, 1992.

[5] O. M. Grasselli. A canonical decomposition of linear periodic discrete-time systems. Int. J. Control, 40:201-214, 1984.

[6] O. M. Grasselli and S. Longhi. Pole-placement for nonreachable periodic discrete-time systems. Math. Control Signals Syst., 4:439-455, 1991.

[7] U. Helmke and E. I. Verriest. Structure and parametrization of periodic linear systems. SIAM J. Matrix Anal. Appl. (submitted).

[8] R. A. Meyer and C. S. Burrus. A unified analysis of multirate and periodically time-varying digital filters. IEEE Trans. Circuits and Systems, 22:162-168, 1975.

[9] B. Park and E. I. Verriest. Canonical forms for discretetime periodically time varying systems and a control application. Proc. of CDC'89, Tampa, pp. 1220-1225, 1989.

[10] P. Van Dooren and M. Verhaegen. On the use of unitary state-space transformations, vol. 47 of Special Issue of Contemporary Mathematics in Linear Algebra and Its Role in Systems Theory, Amer. Math. Soc., Providence, R.I., 1985.

[11] A. Varga. Numerically stable algorithm for standard controllability form determination. Electron. Lett., 17:74-75, 1981.

[12] A. Varga. Computation of irreducible generalized statespace realizations. Kybernetika, 26:89-106, 1990.

[13] A. Varga. Balancing related methods for minimal realization of periodic systems. Systems \& Control Lett., 36:339-349, 1999.

[14] A. Varga. Computation of transfer functions matrices of periodic systems. Proc. of CDC'2002, Las Vegas, Nevada, 2002.

[15] A. Varga and P. Van Dooren. Computational methods for periodic systems - an overview. Proc. of IFAC Workshop on Periodic Control Systems, Como, Italy, pp. 171-176, 2001.

[16] A. Varga and P. Van Dooren. Computation of zeros of periodic systems. Proc. of CDC'2002, Las Vegas, Nevada, 2002.

[17] J. H. Wilkinson. The Algebraic Eigenvalue Problem. Oxford University Press, 1965. 